\title{
Eficiencia del carbón activado obtenido experimentalmente a partir de residuos agrícolas del Alto Mayo
}

Efficiency of activated carbon obtained experimentally from agricultural residues Alto Mayo

${ }^{1}$ Wagner Colmenares M..${ }^{\mathrm{a}}$ Leonardo Damián S. ${ }^{\mathrm{b}}{ }^{2}$ Yrwin Azabache L. ${ }^{\mathrm{a}}{ }^{2}$ Yeltsin Terrones V.

\section{RESUMEN}

Determina la eficiencia del carbón activado obtenido experimentalmente a partir de residuos agrícolas del Alto Mayo. La población del presente trabajo de investigación viene a ser todos los residuos agrícolas conformado por cáscaras de coco, café y cacao. La muestra fue $1 \mathrm{~kg}$ de cáscara de coco extraída de la provincia de Rioja, $1 \mathrm{~kg}$. de cáscara de café extraída del distrito de Yantaló y $1 \mathrm{~kg}$. de cáscara de cacao extraída del distrito de Jepelacio. Se realizó un análisis de las características físicoquímicas de muestras de coco, cacao y cáscaras de café (porcentaje de humedad, carbono fijo, cenizas totales, porosidad y materia volátil), y se evaluó la temperatura de carbonización-activación (con rangos de $550^{\circ} \mathrm{C}, 600^{\circ} \mathrm{C}, 650^{\circ} \mathrm{C}, 700^{\circ} \mathrm{C}, 750^{\circ} \mathrm{C}$ ) en un tiempo de $1 \mathrm{~min}, 1: 30 \mathrm{~min}, 2 \mathrm{~min}, 2: 30 \mathrm{~min}, 3$ min; haciéndose variar los gramos de carbón activado a pesos de $0,5 \mathrm{~g}, 0,75 \mathrm{~g}, 1,0 \mathrm{~g}, 1,25 \mathrm{~g}, 1,5 \mathrm{~g}$. El carbón activado obtenido de la cáscara de café a una temperatura de $650^{\circ} \mathrm{C}$ resultó ser el más eficiente (96,32\%), en comparación a los obtenidos de las cáscaras de cacao y coco, en los que su eficiencia fue menor.

Palabras clave: Eficiencia de carbón, carbón activado, azul de metileno.

\begin{abstract}
Determines the efficiency of activated carbon obtained experimentally from agricultural residues Alto Mayo. The population of this research becomes all agricultural wastes consisting coconut shells, coffee and cacao. The sample was $1 \mathrm{~kg}$ coconut shell taken from the province of Rioja, $1 \mathrm{~kg}$ coffee husk removed Yantalo district and $1 \mathrm{~kg}$ of cocoa husk removed Jepelacio district. For this analysis the samples physicochemical coconut, cocoa and coffee husks (moisture, fixed carbon, total ash, volatile matter and porosity) characteristics was performed, and the carbonization temperature-activation was evaluated (with ranges $550^{\circ} \mathrm{C}, 600^{\circ} \mathrm{C}, 650^{\circ} \mathrm{C}, 700^{\circ} \mathrm{C}, 750^{\circ} \mathrm{C}$ ) in a time of $1 \mathrm{~min}, 1: 30 \mathrm{~min}, 2 \mathrm{~min}, 2: 30 \mathrm{~min}, 3$ min; being varied grams of activated charcoal to weights of $0,5 \mathrm{~g}, 0,75 \mathrm{~g}, 1,0 \mathrm{~g}, 1,25 \mathrm{~g}, 1,5 \mathrm{~g}$. Which indicates that at a temperature of $650^{\circ} \mathrm{C}$, coffee husk has $96,32 \%$ adsorption in methylene blue solution compared to residues of coconut and cacao, accordingly coffee husk is more efficient adsorption in the removal of methylene blue.
\end{abstract}

Keywords: Efficiency coal, activated carbon y methylene blue.

\footnotetext{
'Universidad Nacional de Jaén. Cajamarca, Perú

${ }^{2}$ Universidad Nacional San Martín. Tarapoto, Perú

${ }^{\mathrm{a}}$ Ingeniero Químico, ${ }^{\mathrm{b}}$ Licenciado en Matemática, ${ }^{\mathrm{c}}$ Ingeniero Ambiental
} 


\section{INTRODUCCIÓN}

En el Alto Mayo existen cultivos de coco, café, cacao, entre otros. Esto trae como consecuencia que la generación de residuos agrícolas sea abundante y como consecuencia se genera un impacto ambiental, además de ser el hábitat apropiado para la proliferación de vectores de diferentes plagas o enfermedades.

En los cultivos de café, mediante un proceso mecánico se separa la cáscara de la pepa interior. Las cáscaras obtenidas no tienen casi ninguna aplicación y por eso las arrojan, a los ríos, arroyos, a tierras no cultivadas o las queman. La contaminación por las cáscaras de café es tan considerable como la producida por las de coco y cacao, aunque no se puede ver mucho este impacto, porque la producción de café generalmente se concentra en el campo agrícola o en fincas.

El carbón activado es un adsorbente preparado a partir de materiales carbonosos que se caracteriza por poseer una alta superficie interna y una buena distribución de poros, propiedades que le permiten atrapar una gran diversidad de moléculas (Bardález, 2005).

La activación química de residuos lignocelulósicos con ácido fosfórico produce carbones con una estructura porosa bien desarrollada cuyas características dependen fundamentalmente de la relación de impregnación. Las relaciones de impregnación bajas producen carbones con una estructura predominantemente micro poroso, mientras que relaciones de impregnación altas producen carbones con una porosidad ancha y una importante contribución de micro poros anchos y meso poros (García, 2008).

En Cuba existen condiciones para la producción de carbón activado a nivel industrial. De acuerdo con ello, la producción de este material es de altos precios y demanda creciente en el ámbito mundial, deberá estar enfocada, como estrategia fundamental, hacia el sector del mercado, tanto nacional como internacional, vinculado con la protección del medio ambiente (Fernández y Castillo, 2006).

En México se está proponiendo un nuevo tipo de agroindustria que utilice a una materia prima nacional (cáscara de coco) considerada basura, transformándola en una industria sustentable de un material con mayor valor agregado (carbón activado), que genere ingresos económicos a las personas que viven del coco, nuevos empleos y que ayude a reducir la importación de este producto (Luna, González, Gordon y Martín, 2007).

Se sintetizaron carbones activados por activación química de cáscara de castaña obteniéndose materiales con micro y meso porosidad y con áreas superficiales entre 1176$1434 \mathrm{~m}^{2} / \mathrm{g}$ y al incrementar la razón de impregnación y la temperatura de activación en los carbones preparados, aumentó su grado de porosidad (Paredes, 2011).

Los tratamientos que mejor decoloran para las variedades de coco enano y gigante es utilizando ácido fosfórico a 50 ppm., sobresaliendo en un $0,6 \%$ más eficiente respecto al otro agente activante (ácido sulfúrico), a la misma concentración (Bárdales, 2005).

Este trabajo de investigación tiene como finalidad determinar la eficiencia del carbón activado obtenido experimentalmente a partir de residuos agrícolas del Alto Mayo.

\section{MATERIALES Y MÉTODOS Área de estudio}

El área de estudio abarca las provincias de Rioja y Moyobamba, ambas provincias se localizan 
en la Regíon de San Martin, ubicando detalladamente se intervino en la ciudad de Rioja, el distrito de Yantaló y el distrito de Jepelacio ambos pertenecientes a la provincia de Moyobamba. La población del presente trabajo de investigación viene a ser todos los residuos agrícolas conformado por cáscaras de coco, café. La muestra fue $1 \mathrm{~kg}$ de cáscara de coco extraída de la provincia de Rioja, $1 \mathrm{~kg}$ de cáscara de café extraída del distrito de Yantaló y $1 \mathrm{~kg}$ de cáscara de cacao extraída del distrito de Jepelacio. El tipo de investigación de acuerdo a la orientación será aplicativa y de acuerdo a la técnica de contratación será explicativa. Estadísticamente se aplicó el análisis de varianza (ANAVA). También se utilizó la prueba estadística de DUNCAN al 5\%, con 95\% de confiabilidad y el diseño completamente aleatorizado (DCA) la cual se aplicó para las muestras de carbón activado obtenidas a partir de los residuos agrícolas del Alto Mayo tales como: cáscara de coco, cáscara de café y cáscara de cacao respectivamente.

\section{DETERMINACIÓN DE LA HUMEDAD}

$$
\text { Humedad }=\frac{\mathrm{Pi}-\mathrm{Pf}}{\mathrm{Pi}} \times 100
$$

Donde: Pi : Peso inicial

Pf : Peso final

\section{DETERMINACIÓN DE LAS CENIZAS TOTALES}

Cenizas Totales $=\frac{\mathrm{P} 2}{\mathrm{P} 1} \times 100$

Donde: P1 : Peso final

$\mathrm{P} 2$ : Peso inicial

3. DETERMINACIÓN DE CARBONO FIJO Carbono fijo $=\frac{\mathrm{P} 2}{\mathrm{P} 1} \times 100$

\section{DETERMINACIÓN DE LA POROSIDAD}

\section{A) Procedimiento:}

La porosidad de la materia prima, se calculó de la siguiente manera:

1. Se colocó el material en una probeta, se midió su volumen.

2. Luego se colocó agua hasta el tope del material.

3. Se midió el volumen agregado y se dividió entre el volumen ocupado por el material.

4. Se hicieron 5 repeticiones para cada muestra (cáscara de coco, café y cacao), posteriormente de promedió.

\section{DETERMINACIÓN DE LA MATERIA VOLÁTIL.}

$$
\% \mathrm{Mv}=100-\% \mathrm{C}-\% \text { Ceniza }-\% \mathrm{H}
$$

\section{Dónde \\ $\% \mathrm{Mv}=$ porcentaje de materia volátil. \\ $\% \mathrm{C} \quad=$ porcentaje de carbono fijo. \\ $\%$ Ceniza $=$ porcentaje de ceniza. \\ $\% \mathrm{H} \quad=$ porcentaje de humedad.}

El poder adsorbente del carbón activado puede ser evaluado por su capacidad de decolorar sustancias que contienen colorantes o tintes, de esta manera se tiene una idea de la cantidad de centros activos que éste posee.

Haciendo uso del espectrofotómetro se realiza la lectura de cuanto ha adsorbido el carbón activado frente a la solución de azul de metileno. Mediante este método se determina que porcentaje de la solución ha sido adsorbida por el carbón activado.

La solución de azul de metileno utilizada fue de 1 ppm, la cual, el volumen estable de la solución es de $20 \mathrm{ml}$. 


\section{RESULTADOS}

Tabla 1. Análisis de varianza para el porcentaje de adsorción del carbón activado de cáscara de coco

\begin{tabular}{|c|c|c|c|c|c|}
\hline Fuente de & $\begin{array}{c}\text { Suma de } \\
\text { Variación } \\
\text { Cuadrados } \\
\text { (SS) }\end{array}$ & $\begin{array}{c}\text { Grados } \\
\text { de } \\
\text { Libertad } \\
(\mathrm{gl})\end{array}$ & $\begin{array}{c}\text { Cuadrado } \\
\text { Medio } \\
\text { (MS) }\end{array}$ & $\begin{array}{c}\mathrm{F} \\
\text { calculada }\end{array}$ & $\begin{array}{c}\mathrm{F} \\
\text { tabular }\end{array}$ \\
\hline Temperaturas & 7106,6536 & 4 & 1776,6634 & 112,329 & 2,87 \\
\hline Error & 316,332 & 20 & 15,8166 & & \\
\hline Total & 7422,9856 & 24 & & & \\
\hline
\end{tabular}

\section{Interpretación:}

Se muestran los resultados del análisis de varianza para el porcentaje de adsorción del carbón activado de cáscara de coco, obtenidos producto de la aplicación de las fórmulas estadísticas (suma de cuadrados, cuadrado medio y prueba $\mathrm{F}$ de Fisher-Snedecor) para la verificación de la hipótesis, obteniéndose un valor calculado de $F_{c}=112,329$ y un valor tabular de $\mathrm{F}_{\mathrm{ti}}=0,17$ y $\mathrm{F}_{\mathrm{ts}}=87,2$ (obtenido de la tabla de probabilidad de la distribución $\mathrm{F}$ de
Fisher - Snedecor con 4 y 20 grados de libertad y un nivel de significancia del 5\%), observándose que el valor calculado es mayor que el valor tabular derecho, el cual permite que la hipótesis nula $\mathrm{H}_{0}: \mathrm{m}_{1}=\mathrm{m}_{2}=\mathrm{m}_{3}=\mathrm{m}_{4}=\mathrm{m}_{5}$ se ubique dentro de la región de rechazo, por consiguiente se decide aceptar la hipótesis de investigación $\mathrm{H}_{1}: \mathrm{m}_{1}{ }^{1} \mathrm{~m}_{2}{ }^{1} \mathrm{~m}_{3}{ }^{1} \mathrm{~m}_{4}{ }^{1} \mathrm{~m}_{5}$. Significando que al $95 \%$ de confianza, existe diferencia significativa en el porcentaje de adsorción respecto a las temperaturas que es sometida a la cáscara de coco.

\section{DETERMINACIÓN DE LA TEMPERATURA ÓPTIMA PARA EL CARBÓN ACTIVADO DE CÁSCARA DE COCO}

a) Determinación de la desviación estándar de la media:

$$
S_{\bar{x}}=\sqrt{\frac{C M_{e}}{r}}=\sqrt{\frac{316,332}{5}}=7,954
$$

b) Determinación de la amplitud del límite de significación de Duncan $A L S(D)=A E S(D) S_{\bar{x}}$

\begin{tabular}{l|llll}
$\mathrm{p}$ & 2 & 3 & 4 & 5 \\
\hline AES(D) & 2,95 & 3,097 & 3,19 & 3,255 \\
$S_{\bar{x}}$ & 7,954 & & & \\
\hline ALS(D) & 23,4643 & 24,633538 & 25,37326 & 25,89027
\end{tabular}


c) Matriz de diferencias

\begin{tabular}{l|lllll} 
& $\mathrm{T}_{1}$ & $\mathrm{~T}_{2}$ & $\mathrm{~T}_{3}$ & $\mathrm{~T}_{5}$ & $\mathrm{~T}_{4}$ \\
& 39,92 & 53,84 & 60,36 & 80,42 & 85,34 \\
\hline $\mathrm{T}_{1}=39,92$ & - & 13,92 & 20,44 & 40,5 & $45,42^{*}$ \\
$\mathrm{~T}_{2}=53,84$ & - & - & 6,52 & 26,58 & 31,5 \\
$\mathrm{~T}_{3}=60,36$ & - & - & - & 20,06 & 24,98 \\
$\mathrm{~T}_{5}=80,42$ & - & - & - & - & 4,92 \\
$\mathrm{~T}_{4}=85,34$ & - & - & - & - & - \\
\hline ALS(D) & & 23,4643 & 24,633538 & 25,37326 & 25,89027
\end{tabular}

$(*)$ Diferencia significativa al 5\%

\section{d) Determinación de la temperatura óptima: $\mathbf{T}_{4}$}

La temperatura óptima que debe ser sometido la cáscara de coco para la obtención del carbón activado es de $700{ }^{\circ} \mathrm{C}$.

Tabla 2. Análisis de varianza para el porcentaje de adsorción del carbón activado de cáscara de café.

\begin{tabular}{|c|c|c|c|c|c|}
\hline $\begin{array}{c}\text { Fuente de } \\
\text { Variación }\end{array}$ & $\begin{array}{c}\text { Suma de } \\
\text { Cuadrados } \\
(\mathrm{SS})\end{array}$ & $\begin{array}{c}\text { Grados } \\
\text { de } \\
\text { Libertad } \\
(\mathrm{gl})\end{array}$ & $\begin{array}{c}\text { Cuadrado } \\
\text { Medio } \\
(\mathrm{MS})\end{array}$ & $\begin{array}{c}\mathrm{F} \\
\text { calculada }\end{array}$ & $\begin{array}{c}\text { F } \\
\text { tabular }\end{array}$ \\
\hline Temperaturas & 2659,008 & 4 & 664,752 & 433,4585 & 2,87 \\
\hline Error & 30,672 & 20 & 1,5336 & & \\
\hline Total & 2689,68 & 24 & & & \\
\hline
\end{tabular}

El cuadro muestra los resultados del análisis de varianza para el porcentaje de adsorción del carbón activado de cáscara de café, obtenidos producto de la aplicación de las fórmulas estadísticas (prueba F de Fisher-Snedecor) para la verificación de la hipótesis, obteniéndose un valor calculado de $F_{c}=433,4585$ y un valor tabular de $\mathrm{F}_{\mathrm{ti}}=0,17$ y 2,87 (obtenido de la tabla de probabilidad de la distribución $\mathrm{F}$ de FisherSnedecor con 4 y 20 grados de libertad y un nivel de significancia del 5\%), observándose que el valor calculado es mayor que el valor tabular derecho, el cual permite que la hipótesis nula $\mathrm{H}_{0}: \mu_{1}=\mu_{2}=\mu_{3}=\mu_{4}=\mu_{5}$ se ubique dentro de la región de rechazo, por consiguiente se decide aceptar la hipótesis de investigación $\mathrm{H}_{1}: \mu_{1} \neq \mu_{2} \neq$ $\mu_{3} \neq \mu_{4} \neq \mu_{5}$. Significando que al $95 \%$ de confianza, existe diferencia significativa en el porcentaje de adsorción respecto a las temperaturas que es sometida la cáscara de café. 


\section{TEMPERATURAÓPTIMA PARA ELCARBÓN ACTIVADO DE CÁSCARA DE CAFÉ}

a) Determinación de la desviación estándar de la media:

$$
S_{\bar{x}}=\sqrt{\frac{C M_{e}}{r}}=\sqrt{\frac{30,672}{5}}=2,477
$$

b) Determinación de la amplitud del límite de significación de Duncan $A L S(D)=A E S(D) S_{\bar{s}}$

\begin{tabular}{l|llll}
$\mathrm{p}$ & 2 & 3 & 4 & 5 \\
\hline AES(D) & 2,95 & 3,097 & 3,19 & 3,255 \\
$S_{\bar{x}}$ & 2,477 & & & \\
\hline ALS(D) & 7,30715 & 7,671269 & 7,90163 & 8,062635
\end{tabular}

c) Matriz de diferencias

\begin{tabular}{|c|c|c|c|c|c|c|}
\hline & & $\begin{array}{l}T_{5} \\
63,62\end{array}$ & $\begin{array}{l}\mathrm{T}_{1} \\
69,62\end{array}$ & $\begin{array}{l}\mathrm{T}_{2} \\
78,74\end{array}$ & $\begin{array}{l}\mathrm{T}_{4} \\
88,66\end{array}$ & $\begin{array}{l}\mathrm{T}_{3} \\
89,86\end{array}$ \\
\hline $\mathrm{T}_{5}$ & $=$ & & & & & \\
\hline 63,62 & & - & 6 & 15,12 & 25,04 & $26,24 *$ \\
\hline $\mathrm{T}_{1}$ & $=$ & & & & & \\
\hline 69,62 & & - & - & 9,12 & 19,04 & 20,24 \\
\hline $\mathrm{T}_{2}$ & $=$ & & & & & \\
\hline 7874 & & - & - & - & 9,92 & 11,12 \\
\hline $\mathrm{T}_{4}$ & $=$ & & & & & \\
\hline 88,66 & & - & - & - & - & 1,2 \\
\hline $\mathrm{T}_{3}$ & $=$ & & & & & \\
\hline 89,86 & & - & - & - & - & - \\
\hline ALS(D) & & & 7,31 & 7,67 & 7,9 & 8,06 \\
\hline
\end{tabular}

(*) Diferencia significativa al 5\%

d) Determinación de la temperatura óptima: $\mathbf{T}_{3}$

La temperatura óptima que debe ser sometido la cáscara de café para la obtención del carbón activado es de $650^{\circ} \mathrm{C}$.

Tabla 3. Análisis de varianza para el porcentaje de adsorción del carbón activado de cáscara de cacao

\begin{tabular}{|c|c|c|c|c|c|}
\hline $\begin{array}{c}\text { Fuente de } \\
\text { Variación }\end{array}$ & $\begin{array}{c}\text { Suma de } \\
\text { Cuadrados } \\
(\mathrm{SS})\end{array}$ & $\begin{array}{c}\text { Grados de } \\
\text { Libertad (gl) }\end{array}$ & $\begin{array}{c}\text { Cuadrado } \\
\text { Medio (MS) }\end{array}$ & F calculada & F tabular \\
\hline Temperaturas & 5094,9816 & 4 & 1273,7454 & 102,796013 & 2,87 \\
\hline Error & 247,82 & 20 & 12,391 & & \\
\hline Total & 5342,8016 & 24 & & & \\
\hline
\end{tabular}


Según el cuadro, se muestra los resultados del análisis de varianza para el porcentaje de adsorción del carbón activado de cáscara de cacao, obtenidos producto de la aplicación de las fórmulas estadísticas (prueba $\mathrm{F}$ de FisherSnedecor) para la verificación de la hipótesis, obteniéndose un valor calculado de $\mathrm{F}_{\mathrm{c}}=102,796013 \mathrm{y}$ un valor tabular de $\mathrm{F}_{\mathrm{ti}}=0,17 \mathrm{y}$ $\mathrm{F}_{\mathrm{ts}}=87,2$ (obtenido de la tabla de probabilidad de la distribución F de Fisher - Snedecor con 4 y 20 grados de libertad y un nivel de significancia del $5 \%$ ), observándose que el valor calculado es mayor que el valor tabular derecho, el cual permite que la hipótesis nula $\mathrm{H}_{0}: \mu_{1}=\mu_{2}=\mu_{3}=\mu_{4}$ $=\mu_{5}$ se ubique dentro de la región de rechazo, por consiguiente se decide aceptar la hipótesis de investigación $\mathrm{H}_{1}: \mu_{1} \neq \mu_{2} \neq \mu_{3} \neq \mu_{4} \neq \mu_{5}$. Significando que al 95\% de confianza, existe diferencia significativa en el porcentaje de adsorción respecto a las temperaturas que es sometida la cáscara de cacao.

\section{DISCUSIÓN}

El carbón activado es un término general que se aplica a toda una serie de productos derivados de materiales carbonosos; es un material amorfo que presenta un área superficial excepcionalmente alta y se caracteriza por tener una proporción de micro poros (poros menores que 2 nanómetros). Estas características le confieren propiedades adsorbentes excepcionales que pueden ser aprovechadas en diferentes áreas. La preparación de carbón activado se realiza a partir de materia orgánica en su mayoría de origen vegetal, por carbonización o por acción de sustancias químicas (agentes químicos) (Rodríguez, 2005).

En el Alto Mayo se practican los cultivos de coco, café, cacao, la cual se consideran como fuentes de ingreso. Durante el proceso de cosecha se genera una gran cantidad de residuos agrícolas que son arrojados y no son aprovechados por los agricultores, esto como consecuencia genera impactos ambientales como la lixiviación, problemas estéticos del medio, además la acumulación de estos se considera como un medio apropiado para la proliferación de distintos vectores de enfermedades, por lo que implicaría serios problemas sanitarios (Bardález, 2005).

En la presente investigación se centró en una variable que es la temperatura. Esta variable es muy importante ya que influye sobre la naturaleza en la formación del centro activo del carbón y en la formación de los poros del carbón activado (macro poros, meso poros y micro poros). A partir de este análisis, se identificó cual es la temperatura óptima (eficiencia) en donde se produce una mayor adsorción de la solución de azul de metileno.

Al realizar el análisis de la efíciencia del carbón activado (porcentaje de adsorción de la solución de azul de metileno), se ha demostrado que el carbón activado de la cáscara de coco, café y cacao, tienen diferente porcentaje de adsorción, debido a la cantidad y distribución de poros que presenta cada uno de ellos, por lo que se ha observado que a mayor distribución de poros del carbón activado entonces va haber mayor porcentaje de adsorción.

\section{CONCLUSIONES}

Durante el proceso de adsorción, se determinó que la eficiencia del carbón activado de la cáscara de coco es a una temperatura de $700^{\circ} \mathrm{C}$ $(85,34 \%)$, tiempo de contacto del carbón activado con la solución de azul de metileno de $1: 30 \mathrm{~min}(85 \%)$ y agregando $0.5 \mathrm{~g}(85,6 \%)$. 
La eficiencia del carbón activado de la cáscara de café es a una temperatura de $650^{\circ} \mathrm{C}(89,86 \%)$, tiempo de contacto del carbón activado con la solución de azul de metileno de $2 \min (76 \%)$ y agregando $1 \mathrm{~g}(85.6 \%)$.

La eficiencia del carbón activado de la cáscara de café es a una temperatura de $650^{\circ} \mathrm{C}(96,32 \%)$, tiempo de contacto del carbón activado con la solución de azul de metileno de $1 \min (88,1 \%)$ y agregando $0,5 \mathrm{~g}(89.4 \%)$.

De acuerdo al análisis estadístico realizado, se concluye que la temperatura óptima que debe ser sometido la cáscara de coco para la obtención del carbón activado es de $700^{\circ} \mathrm{C}$, la cáscara de café a $650^{\circ} \mathrm{C}$ y finalmente la cáscara de cacao a $650^{\circ} \mathrm{C}$.

Se concluye que entre los tres residuos agrícolas, el carbón activado obtenido de la cáscara de café presenta mayor adsorción en la remoción del azul de metileno, con una eficiencia de 96,32\%.

\section{AGRADECIMIENTOS}

A la Universidad Nacional de Jaén por darme la oportunidad de publicar esta investigación.

\section{REFERENCIAS BIBLIOGRÁFICAS}

Bardález V, A. 2005. “Obtención química de carbón activado a partir del endocarpo de coco (Cocos nucifera) en la región San Martín". Tarapoto- Perú.

Fernández R. A. y C. R. Castillo E. 2006. "Aspectos industriales de la producción de carbón activado y sus aplicaciones en la mitigación ambiental". La Habana-Cuba.

García M, J. B. 2008. "Catalizadores carbonosos obtenidos mediante activación química de residuos lignocelulósicos”. Málaga.
Luna D, A. Gonzáles, M. Gordon y N. Martín. 2007. "Obtención de carbón activado a partir de la cáscara de coco". México.

Paredes D, A. L. 2011. "Estudio de la adsorción de compuestos aromáticos mediante carbón activado preparado a partir de la cáscara de castaña”. Lima-Perú.

Rodríguez, F. 2005. "Carbón activado: estructura, preparación y aplicaciones", Revista Uniandes, pág. 66-6. Granada.

\section{Correspondencia:}

Wagner Colmenares Mayanga

Calle Huáscar $\mathrm{N}^{\circ} 713$ - distrito de Olmos Lambayeque, Perú.

wcolmenaresm@hotmail.com 